energetischen System anzusehen, der zu einem ,hochdispersen Verteilungszustand" der Kolloide führt. Ob dadurch wirklich bereits, wie BIERICH schreibt, ,an Stelle der verschwommenen, teleologischen Begriffe des formativen, entzündlichen und blastogenen Reizes einerseits und an Stelle des unbestimmten Begriffes der Zelle andererseits" gut definierbare Vorstellungen gewonnen werden, dürfte wohl noch recht zweifelhaft sein.

Man kann weiter gegen die Verwendung der Erfahrungen der Tierversuche auf die menschliche Pathologie noch den Einwand machen, daß beim Menschen doch nur ausnahmsweise die schädigenden Stoffe in ähnlicher Konzentration und in ähnlicher Zeitdauer einwirkten, wie in den Versuchen, und daß z. B. unter den Teer- und Paraffinarbeitern die einen nach sehr langer Einwirkung nicht an Krebs, andere dagegen nach kürzerer Einwirkung an Krebs erkrankten. Und damit würde dann wieder die Konstitutions- und Dispositionsfrage aufgeworfen werden. Fibiger hat schon die Meinung ausgesprochen, daß beim Spiropterenkrebs der Ratten der Inzucht eine gewisse prädisponierende Rolle zuzukommen scheine; und sicherlich sind derartige Umstände auch in der menschlichen Geschwulstpathologie bedeutungsvoll, aber doch nur in dem Sinne, daß sie zur Erklärung herangezogen werden müssen für die Beantwortung der Frage, warum unter den gleichen Bedingungen der eine früher, der andere später, der dritte gar nicht an Krebs erkrankt.

\section{DER OSMOTISCHE DRUCK, SEINE BEDEUTUNG UND SEINE REGULATION IM TIERKÖRPER.}

Von

\author{
Prof. Dr. EmIL ReIss in Frankfurt a. M.
}

Begriffsbestimmung und Gesetze. Der osmotische Druck ist eine Vergleichsgröße. Eine Lösung, für sich allein betrachtet, hat keinen osmotischen Druck. Ein osmotischer Druck entsteht, wenn zwei verschieden konzentrierte Lösungen durch ein Medium voneinander getrennt sind, welches für das Lösungsmittel durchgängig, für die gelöste Substanz aber undurchgängig ist. Wenn wir z. B. ein Gefäß durch eine für Zucker undurchlässige Membran in zwei Hälften teilen und die eine Seite mit Wasser, die andere Seite mit einer I proz. Zuckerlösung in gleicher Höhe füllen, so diffundiert das Wasser durch die Membran in die Zuckerlösung hinein, so daß diese bei entsprechender Versuchsanordnung bis zur Höhe von mehr als $7 \mathrm{~m}^{\mathbf{1}}$ ) ansteigt. Somit ist an die Stelle des osmotischen Drucks ein statischer Druckunterschied getreten.

Die osmotische Wirksamkeit einer gelösten Substanz hängt ab von der in der Gewichtseinheit enthaltenen Zahl der Moleküle. Aquimolekulare Lösungen haben den gleichen osmotischen Druck. Bei gleichprozentigen Lösungen ist der osmotische Druck um so höher, je kleiner das Molekulargewicht, je größer also die Zahl der Moleküle. Außerdem hängt der osmotische Druck einer gelösten Substanz ab von ihrer Dissoziierbarkeit, da jedes Ion die gleiche osmotische Wirkung hat wie das ganze Molekül. Daher haben die Elektrolyte, $z$. B. verdünnte Salzlösungen, einen nahezu doppelt so großen osmotischen Druck als sich aus ihrem Molekulargewicht errechnen läBt. Substanzen mit besonders hohem Molekulargewicht und geringer Dissoziierbarkeit, wie die Eiweißkörper, verursachen keinen mit den gewöhnlichen Methoden erkennbaren osmotischen Druck.

Die osmotischen Druckwerte, die aus diesen Gesetzen berechnet werden, gelten jedoch nur für den Fall idealer semipermeabler Membranen, d. h. solcher, die für das Lösungsmittel leicht durchlässig, für die gelöste Substanz aber völlig undurchlässig sind. Die meisten Membranen weichen von diesem Idealzustand mehr oder minder ab. Die Art der Membran ist also von grundlegender Bedeutung für den zur tatsächlichen Auswirkung Kommenden Druck. Würde die

1) Der genaue Wert entspricht innerhalb der Temperaturen von $\mathbf{1 3}, 2^{\circ}$ bis $\mathbf{1} 6, \mathbf{1}^{\circ} \mathrm{C}$ einer Quecksilbersäule von $53,8 \mathrm{~cm}$, d. i. $732 \mathrm{~cm}$ Wasser (PFEFFER, Osmotische UnterQuecksilbersäule von 53,8
suchungen, Leipzig 1877 ).
Membran, in dem obenangeführten Beispiel, aus Glas bestehen, also auch für Wasser praktisch undurchlässig sein, so wäre ein osmotischer Druckunterschied nur theoretisch vorhanden, denn tatsächlich würden $\mathrm{zu}$ seiner Ausgleichung unendlich lange Zeiten erforderlich sein. Wäre umgekehrt die Membran so dünn, daß der Zucker fast ebenso schnell durchträte wie Wasser, so würden die Bedingungen für einen größeren Druckunterschied nicht gegeben sein. Diese Tatsachen sind auch biologisch von großer, bisher meist verkannter Wichtigkeit, denn darauf beruht es, daß im Organismus fast nie der ganze rechnerisch ermittelte osmotische Druckunterschied zweier Lösungen zum Austrag kommt. Ein hochkonzentrierter Urin kann längere Zeit in der Blase liegen, ohne mit dem Blut in Beziehung zu treten. Die dicke, kaum mehr zum Wasseraustausch befähigte, Blasenwand läßt die theoretisch vorhandene Druckdifferenz in kurzer Zeit nicht zum Austrag kommen. Sind dagegen zwei verschieden konzentrierte Flüssigkeiten nur durch die Hülle zarter Gewebszellen voneinander getrennt, so wird auch dem Durchtritt osmotisch wirksamer Substanzen nur wenig Widerstand entgegengesetzt, in diesem Falle können hohe Druckdifferenzen gar nicht entstehen. Es ist also für den Flüssigkeitsaustausch im Organismus nicht gleichgültig, ob zwei verschieden konzentrierte Lösungen durch dicke Epithelschichten oder etwa durch die Bowmansche Membran der Glomeruli voneinander getrennt sind. Ideale halbdurchlässige Membranen kommen im Tierkörper kaum vor. Wir dürfen also im Organismus aus Konzentrationsdifferenzen nicht ohne weiteres osmotische Drucke von einem Grade herleiten, der nur als höchster Grenzwert $z$. B. mittels der Niederschlagsmembranen in der PFEFFERschen Tonzelle erreicht wird.

Der osmotische Druck als Triebkraft. Als man den osmotischen Druck im Tierkörper zu messen anfing, überraschte zunächst am meisten der Gegensatz zwischen dem gleichmäßigen osmotischen Druck von Blut und Gewebssäften einerseits und den hiervon wesentlich abweichenden und schwankenden Werten, die man für Urin, für den Inhalt von Magen und Darm sowie für einige andere Se- und Exkrete fand. Man hielt diese groben Differenzen für den Ausdruck einer Energiespannung und sah darin die hauptsächliche physiologische Bedeutung des osmotischen Druckes. Man glaubte nun endlich die langgesuchte Triebkraft für verschiedene bislang unerklärte Vorgänge wie Drüsensekretion, Darmresorption, Harnbereitung u. a. gefunden zu haben. Man stellte mathematische Formeln auf, aus denen sich die Umwandlung des osmotischen Drucks in die physiologischen Energieformen ergeben und das Geheimnis der Kraftquellen des Lebens erklären sollte. Es ist nicht $Z$ weck dieser kurzen Darlegungen, alle Theorien $z u$ besprechen, die in jener Zeit entstanden sind und deren Reste noch heute gelegentlich auftauchen. Nur an einigen Beispielen sollen die prinzipiellen Seiten der Frage beleuchtet werden.

Vor allem war es die Harnbereitung oder mindestens Teile dieses Vorganges, die man aus der Differenz des osmotischen Druckes zwischen Blut und Urin energetisch zu erklären suchte. Es bedarf nicht des Experimentes, vielmehr zeigen. schon einfache Uberlegungen, daß der osmotische Druck als alleinige oder nur Haupttriebkraft durchaus nicht zur Erklärung der physiologischen Erscheinungen der Nierentätigkeit gen ügt. Folgen wir der üblichen Vorstellung, daß in den Glomerulis primär eine sehr verdünnte Lösung abgeschieden wird, und daß diese hinterher - etwa in den Harnkanälchen - durch Osmose an gelösten Substanzen angereichert wird, indem Wasser in die Blutbahn zurücktritt, so könnten wir die Bildung eines hypotonischen oder bestenfalls blutisotonischen Urins erklären, niemals aber die Abscheidung einer hypertonischen Flüssigkeit. Wenn man aber bedenkt, $\mathrm{da} B$ bei Menschen und höheren Tieren der Urin je nach der zugeführten festen und flüssigen Nahrung bald hypotonisch, bald isotonisch, weitaus am häufigsten aber hypertonisch ist, so ergibt sich, daß hier andere Trieblrräfte als der osmotische Druck am Werk sein müssen. Der Endeffekt der Arbeit, welche die Niere bei Herstellung eines hypertonischen. Urins leistet, besteht darin, daB sie dem Blut gelöste Substanzen 
entzieht, ohne ihm auch die osmotisch entsprechende Menge Wasser zu rauben. Die Nierentätigkeit erzeugt also einen osmotischen Druckunterschied, und es ist nicht umgekehrt, wie man anfangs meinte, die Harnproduktion eine Folge osmotischer Kräfte.

Berechnung der Nierenarbeit. Es muß an dieser Stelle ein an sich geistreiches Rechenexempel erwähnt werden, das ausgedacht war, um die Größe der energetischen Nierenleistung zu ermitteln. Die Tatsache, daß bei der Harnbereitung ein Druckunterschied erzeugt wird, führte DRESER ${ }^{1}$ ) auf den Gedanken, die Arbeitsleistung der Niere aus den osmotischen Wertdifferenzen von Harn und Blut zu berechnen. Er lkam zu folgenden Resultaten: Zur Herstellung von $200 \mathrm{ccm}$ eines Urins vom Gefrierpunkt $-2,3^{\circ}$ aus Blut vom Gefrierpunkt - $0,5^{\circ}$ müsse die Niere eine Arbeit entsprechend etwa $37 \mathrm{mkg}$ leisten. Daraus würde sich die 24stündige Arbeit der Niere auf etwa 200 mkg berechnen. Mit der maximalen Kraft des menschlichen Muskels verglichen, würde die Leistung der Niere nach DRESER mehr als das 6 fache betragen. Der ProzeB der Wasserausscheidung in den Nieren wird nach DRESER durch die antagonistischen Wirkungen der Sekretion und der Resorption reguliert. Der hierbei auftretende maximale Sekretionsdruck der Niere (im Beispiel einer durstenden Katze) beträgt $49 \mathrm{~m}$ Wasser, der maximale Resorptionsdruck mehr als $500 \mathrm{~m}$ Wasser. Der Blutdruck entspricht dagegen einer Wassersäule von nur etwa $2,72 \mathrm{~m}$, er könnte eine osmotische Differenz zwischen Blut und Harn von höchstens $0,022^{\circ}$ hervorbringen. Diese imposanten Zahlen Dresers eriklären sich - abgesehen von anderen groben Fehlern in der Anlage der Berechnungen - hauptsächlich aus der Voraussetzung, daß die trennenden Membranen in idealer Weise semipermeabel seien, da $B$ also die Nieren stets gegen den höchstmögtichen osmotischen Druck anzukämpfen haben. Da es aber solche, für das Lösungsmittel völlig durchgängige und für die gelösten Substanzen absolut undurchlässige Membranen im Körper nicht gibt, und gerade in den Nieren aus naheliegenden physiologischen Gründen nicht geben kann, sind auch alle diese Berechnungen mit ihren phantastischen Endresultaten wertlos.

Auch für den Vorgang der-Darmresorption hat man versucht, den osmotischen Druck als Triebkraft hinzustellen. Der Uberdruck, den die Ingesta gegenüber Blut und Lymphe besitzen, sollte die Aufnahme von Nahrungsstoffen aus dem Darmlumen in die Gewebe herbeiführen. Diese Anschauung hat sich in der Physiologie merkwürdig lange gehalten. Einfache Uberlegungen zeigen jedoch, daß osmotische Triebkräfte überhaupt nux in zwei Fällen zugunsten der Resorption wirksam sein können, nämlich zur Aufnahme von Wasser aus hypotonischen Lösungen und zur Aufnahme von Salzen und anderen gelösten Substanzen aus hypertonischen Lösungen (itn der Voranssetzung, daß die Membranen auch Salze teilweise durchlassen). In allen anderen Fällen können osmotische Triebkräfte unmöglich in Betracht kommen. Die Richtigkeit dieser theoretischen Gesichtspunkte wurde durch ausgedehnte Versuche von HEIDENHAIN ${ }^{2}$ ) und seitdem durch zahllose weitere Erfahrungen vollauf bestätigt. HEIDENHAIN hat eine in situ belassene Dünndarmschlinge des Hundes an zwei Stellen zugebunden, dann eine bestimmte Lösung eingefüllt, nach verschiedenen Zeitabständen wieder entleert und den Inhalt untersucht. Ich gebe nachstehend einige Versuche HeIdenHains in vereinfachter Darstellung wieder.

Versuchsreithe XI (S. 6oI).

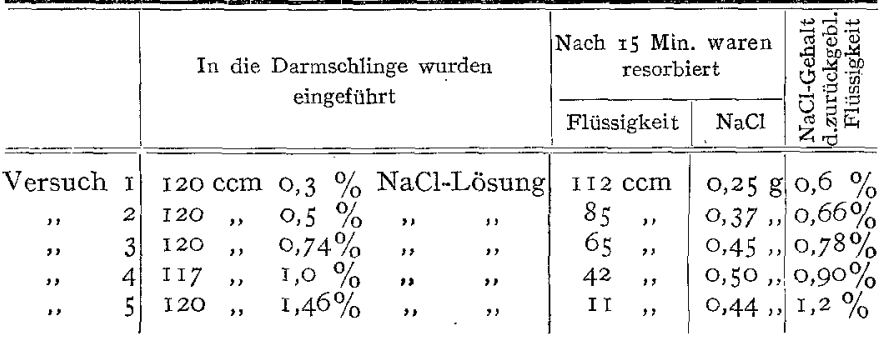

i) Uber Diurese und ihre Beeinflussung durch pharmakologische Mittel. Atch. f. experim. Tathol. u. Pharmakol. 29, 303. I892.

2) Arch, f. d. ges. Physiol. 56, 579. 1894 .
Versuchsreihe XVI (S. 6II).

\begin{tabular}{|c|c|c|c|c|c|}
\hline & & \multicolumn{3}{|c|}{$\begin{array}{c}\text { Von } 80 \mathrm{ccm} \text { einer I,98\%proz. } \mathrm{NaCl} \text {-Lösung } \\
\text { wurden resorbiert }\end{array}$} & \multirow{2}{*}{$\begin{array}{c}\text { NaCl-Gehalt den } \\
\text { zurückgeblieb. } \\
\text { Flüssigkeit }\end{array}$} \\
\hline & & & Flüssigkeit & $\mathrm{NaCl}$ & \\
\hline $\begin{array}{c}\text { Versuch } \\
\qquad, \\
,,\end{array}$ & $\begin{array}{r}\text { I } \\
\text { III }\end{array}$ & $\begin{array}{l}\text { in } 20 \text { Min } \\
, 40 \quad, \\
, 60 \quad,\end{array}$ & $\begin{array}{l}0 \\
\text { I } 7 \\
38 \%\end{array}$ & $\begin{array}{l}0,62 \mathrm{~g} \\
0,89 ", \\
\mathrm{I}, \mathrm{I} 8,2\end{array}$ & $\begin{array}{l}\mathrm{T}, 20 \% \\
\mathrm{I}, 10 \% \\
0,96 \%\end{array}$ \\
\hline
\end{tabular}

Die erste Tabelle zeigt eine Versuchsreihe, in welcher gleiche Mengen verschiedenprozentiger Kochsalzlösungen gleichlange Zeit in der gleichen Darmschlinge belassen wurden (Versuchsreihe XI). Man sieht, daß un so mehr Wasser resorbiert wurde, je verdünnter die Lösung war. Umgekehrt stieg zwar die resorbierte Salzmenge mit der Konzentration, aber nur bis zu einer gewissen Grenze, die in der Gegend der Isotonie der eingeführten Lösung lag (etwa $0,9 \% \mathrm{NaCl}$ ). Aus der letzten Spalte der Tabelle sieht man, daß die im Darmlumen zurückgebliebene Flüssigkeit sich in allen Fällen dem osmotischen Druck des Blutes näherte, indem hypotonische Flüssigkeiten eingedickt, hypertonische verdünnt wurden. Es hatte also zwischen Darminhalt und Gewebe ein Austausch stattgefunden, der sich teils nach den Gesetzen des osmotischen Drucks, teils gegen sie richtete. Aus Versuchsreihe XVI sehen wir, wie sich die Resorption einer Lösung von bestimmter Konzentration bei verschiedener Dauer ihres Aufenthalts im Darm gestaltet. Es ergibt sich, $\mathrm{da} B$ ans einer stark hypertonischen Lösung zunächst nur Salz aufgesaugt wurde, und daß die Resorption von Wasser sich um so schneller vollzog, je mehr sich die Lösung dem osmotischen Druck des Gewebes näherte. Eine deutliche Wasserresorption fand aber schon vorher statt. Aus diesen Versuchen geht zweifelsfrei hervor, daß zwar osmotische und Diffusionsvorgänge im Darm stattfinden, daß sie aber ebensowohl der Resorption entgegenwirken als sie unterstützen können. Es müssen also außer den osmotischen noch andere Kräfte wirksam sein, ja sogar müssen diese die Hauptrolle spielen. Das Bestreben dieser Kräfte geht dahin, den Darminhalt in jedem Falle dem osmotischen Druck des Blutes näher zu bringen. Isotonische Lösungen liefern den aufsaugenden Zellen offenbar die besten Bedingungen für die Resorption der Gesamtlösung (Wasser und osmotisch wirkende Substanzen). Weichen die Lösungen von der Isotonie in extremer Weise $a b$, so reagieren die resorbierenden Elemente derart, daß überhaupt keine Resorption mehr stattfindet, ja daß Flüssigkeit in das Darmlumen hinein ausgeschieden wird, solange bis wieder eine maßvolle Konzentration im Darmlumen hergestellt ist. Es gibt aber auch Substanzen, welche die Darmwand so schädigen, daß die Resorption längere Zeit oder endgültig gelähmt wird (Sulfate, Fluornatrium, Sublimat, Arsen usw.). Und das alles geschieht, obwohl die physikalischen Bedingungen für eine Osmose in solchen Fällen nicht verschlechtert, ja sogar manchmal besonders günstige geworden sind. Ist dagegen der Darminhalt dem Blut isotonisch, fehlt also jede Grundlage für die Mitwirkung osmotischer Kräfte, gerade dann geht die Resorption am besten vonstatten. Das beweist schlagend ein klassischer Versuch Heidenhairs: Hundeblutserum wurde in der Darmschlinge des Hundes völlig resorbiert. Auch klinische Erfahrungen, (z. B. Rectalernährung), zeigen, daß isotonische Lösungen am besten resorbiert werden.

Es ist also durchaus sicher, daß der osmotische Druck als Triebkraft für die Resorption nicht erforderlich ist.

Es ist hier nicht der Ort, auf die verschiedenen Hilfshypothesen einzugehen, mit denen man die Theorie vom osmotischen Druck als Triebkraft zu retten suchte. Sie treffen sämtlich nur für gelegentliche Bedingungen $\mathrm{zu}$, wie sie unter den wechselvollen Verhältnissen des mit den verschiedensten Nahrungsstoffen gefüllten Darmes hin und wieder vorkommen. Aber sie können niemals die einseitig vom Darmlumen ins Gewebe mit großer Geschwindigkeit sich ergießende Flüssigkeitsströmung erklären, die Lösungsmittel und gelöste Substanzen verschiedenster Art und Konzentration mit sich führt. Man vergegenwärtige sich nur einmal die Leistung, die der Organismus vollbringt, wenn 2 oder $31 \mathrm{Bier}$ innerhalb weniger 
Stunden nicht nur völlig aufgesaugt, sondern durch den ganzen Körper durchgeleitet und zu den Nieren wieder ausgeschieden werden. Wie das alles mit der toten Kraft des osmotischen Druckes erklärt werden soll, ist völlig unerfindlich.

Das gleiche gilt für alle übrigen Fälle, wo man komplexe physiologische Vorgänge auf die Triebkraft des osmotischen Druckes zurückführen wollte: für die Bildung der Ductusund der Gewebslymphe, für die Entstehung des Speichels, der Magensalzsäure, der Galle, für die Ausscheidung der Drüsensekrete nach außen u. a. m. Man darf hinzufügen, daß gleiches Versagen allen anderen Spekulationen beschieden sein $\mathrm{muß}$, die grobe physikalische Kräfte, wie Oberflächenspannung, H-Ionenkonzentration, Quellungsdruck, Membranpotentiale usw. für die zweckmäßigen komplexen Funktionen von Zellen oder ganzen Geweben ohne Rücksicht auf deren feinere Konstruktion verantwortlich machen wollen.

Isotonie. Die wahre Bedeutung des osmotischen Drucks ist eine ganz andere. Sie liegt in erster Linie in seiner Konstanz. Der osmotische Druck ist eine jener Bedingungen des inneren Milieus, welche für die Gewebe des höher organisierten Individuums lebensnotwendig sind. Der normale Ablauf der Zel1vorgänge ist bei höheren Tieren an einen bestimmten osmotischen Druck gebunden, der nur innerhalb enger Grenzen schwankt (Isotonie). Blut, Blutplasma, Blutserum, Gewebslymphe, Lumbalflüssigkeit, Kammerflüssigkeit des Auges; Sperma, Fruchtwasser usw. haben unter normalen Verhältnissen einen nahezu lsonstanten und untereinander fast gleichen Wert des osmotischen Drucks. Geringe Abweichungen von dem Niveau, auf welches die hochdifferenzierte Zelle eingestellt ist, kommen bei einer ganzen Reihe von physiologischen Teilvorgängen vor und wirken - nicht als Triebkraft, - sondern als Reiz, welcher die spezifische Funktion der Zelle auslöst. Nur unter krankhaften Verhältnissen können Blut- und Gewebssäfte erheblichere Differenzen von dem normalen osmotischen Druck aufweisen. Dann pflegen aber fast immer Störungen der Zelltätigkeit einzutreten, wie wir das beispielsweise bei Urämie sehen (s. später).

Osmoregulation. Daß der normale Körper mit so großer Zähigkeit die Isotonie seiner verschiedenen Bestandteile festzuhalten vermag, erscheint zunächst wunderbar. Die Erklärung kann nur in dem Vorhandensein eines äußerst feinen Regulationsmechanismus liegen. Wir müssen uns vorstellen, daß ein auch nur im geringsten vom nornalen abweichender osmotischer Druck sofort einen Reiz auf die Zellen ausübt; dieser osmotische Reiz löst komplizierte Reflexvorgänge aus, die mittels $Z$ u- oder Abfuhr von Wasser oder osmotisch wirksamen Bestandteilen den normalen osmotischen Druck wiederherstellen, und zwar sowohl lokal in kestimmten Gewebsbezirken und Organen wie im Gesamtorganismus.

In Analogie mit anderen Regulationsmechanismen ist es wahrscheinlich, daß es sowohl periphere wie zentrale Regelungen des osmotischen Druckes gibt, und daß diese untereinander und mit anderen Vorgängen in Verbindung stehen durch direkte Reizübertragung, durch Nervenverbindung, durch Hormone und vielleicht durch weitere noch unbekannte Vorrichtungen. Die periphere Regulation könnte von jeder Zelle ausgehen. Es spricht. zunächst nichts dagegen, daß jede Körperzelle imstande ist, auf eine Änderung des osmotischen Druckes der umspülenden oder in sie ejndringenden Gewebsflüssigkeit zu reagieren. Denn die Fähigkeiten zur Quellung oder Schrumpfung kommen jedem Protoplasma zu. Und es wäre möglich, daß der hierbei entstehende Reiz sich durch die gewöhnliche Innervation eines jeden Gewebes fortpflanzt. Neuere Unterswchungen sprechen aber in dem Sinne, daß für die periphere osmotische Sensibilität besondere Organe vorgebildet sind. Als solche werden die Vater-Pacinischen Körperchen (Lamellenkörperchen) angesehen. Ihrem Bau zufolge könnten sie auch, wie man früher annahm, als besonders geeignet zur Empfindung von Elastizitätsveränderungen (Drucksinn) erscheinen. Allein es ist nicht recht verständlich, warum sie dann z. B. im Mesenterium so besonders reichlich enthalten sind. Die neuere Auffassung als ,, Schwellsinnorgane“"
[ScHADE $\left.\left.{ }^{1}\right)\right]$ entspricht ebenfalls der histologischen Struktur dieser Körperchen sehr gut. Die zahlreichen ineinandergeschachteiten Lamellen können als besonders fein abgestufte halbdurchlässige Membranen wirken, die jede Konzentrationsänderung mit einer Änderung des interlamellaren Flüssigkeitsvolumens beantworten, so daß eine Vermehrung oder Verminderung des statischen Drucks entsteht, welche sich auf den Innenkolben überträgt und durch dessen Verbindung mit dem sympathischen Nervensystem den Rèiz weiterleitet. (Auf die ganz äußerliche Ahnlichkeit mit einem Osmometer bestimmter Form möchte ich dabei keinen Wert legen.)

Für đie zentrale Regulation des osmotischen Druckes haben neuere Untersuchungen sehr wichtige Unterlagen geliefert. Am Boden des IV. Ventrikels, in der Rautengrube, liegt nahe dem von ClAUDE BERNARD entdeckten Zuckerstich die Stelle des von EckHard gefundenen Salzstiches. Ihre Zerstörung verursacht erhöhte Ausscheidung von Kochsalz und Wasser (Hyperchlorurie und Polyurie) mit oder ohne Erhöhung des Blutkochsalzspiegels. Es gelingt jedoch auch, eine isolierte Vermehrung der Salzausscheidung zu erzielen [Jungmann und Meyer ${ }^{2}$ )]. Die genaue Stelle des Salz- und Wasserstiches liegt nach den Untersuchungen von AsCHNER ${ }^{3}$ ), ferner von Brugsch, Dreser und LEWY4), in Ganglienzellen der Formatio reticularis an der medialen Seite des Corpus restiforme, dorsal vom Facialis- und Seitenstrangkern, in der Nähe des Parotis-Sekretionszentrums. Von den Kernen der Formatio reticularis gehen Verbindungen nach oben zum Zwischenhirn, and zwar zum Boden des III. Ventrikels, dem Hypothalamus (Corpora mammillaria, Tuber cinereum und Infundibulum). Auch die hier befindlichen Zentren beeinflussen, ebenso wie die Sekretion der mit ihnen in Verbindung stehenden Hypophyse, die Wasser- und Kochsalzausscheidung in spezifischer Weise. Wir kennen also eine ganze Reihe von Regulationsstellen für den Wasser- und Salzstoffwechsel. Wenn dieser auch von maßgebender Bedeutung für das $\mathrm{Zu}$ standelsommen der osmotischen Bilanz sein muß, so können wir doch nicht ohne weiteres die Salz- und Wasserzentren mit Zentren des osmotischen Drucks identifizieren, solange eine direkte Beeinflussung des letzteren nicht nachgewiesen ist. Nur die gegenseitigen Beziehungen zwischen Wasserund Salzzentren oder etwa ein besonderes übergeordnetes Zentrum könnte die Regulation des osmotischen Druckes erklären. Deshalb ist es von Wichtigkeit, daß LESCHKE ${ }^{5}$ ) bei polyurischem Diabetes mellitus durch Konzentrationsversuche eine Eindickung des Blutes mit Erhöhung des osmotischen Drucks herbeiführen konnte, und daß er in solchen Fällon histologische Veränderungen in der Zwischenhirnbasis gefunden hat. Wir dürfen es somit als wahrscheinlich betrachten, daß wir in diesen und vielleicht in noch höheren Gehirnteilen wirkliche Zentren des osmotischen Drucks besitzen. Die Wege, auf denen diese Zentren den osmotischen Druck beejnflussen, dürften zu allen Organen führen, die mit der Ausscheidung bzw. Zurückhaltung von Wasser und gelösten Substanzen betraut sind. Wir kennen die sympathischen Bahnen, die im Splanchnicus zu den Nieren laufen und die auf ihrem ganzen Wege, sogar noch innerhalb der Nieren [JUNGManN $\left.{ }^{6}\right)$ ], JUNGMANN und Seyderhelm $\left.{ }^{7}\right)$ ] Reize empfangen können, welche die Harnkonzentration beeinflussen. Auch der Bauchsympathicus beteiligt sich an der Niereninnervation [ASHER und JoSI $\left.\left.{ }^{8}\right)\right]$. AuBerdem gibt es aber auch parasympathische Verbindungen [AsHeR und $\operatorname{PEARCE}^{9}$ ), MAYERIOFER ${ }^{10}$ ), die im Vagus verlaufen und mit den sympathischon in kompliziertestem Wirkungsverhältnis stehen. Zweifellos stehen aber die medullären und cerebralen Zentren

1) 1) ie physik:lische Chemie in der inneren Medizin, Verlag von Steinkopff, $192 x, S .430$. 2) Arch. f. exparim. Pathol, u. Pharmakol. 73, 49, rgr3 und 3o.Kongreb f. inn. Med.Igr3 S. 2 II.

s) Arch. f. d. ges. Physiol. 146

4) Zeitschr. f. experim. Pathol. u. Ther. 21, 358. 1920

5) Disch. m. d. Wochenschr. 1920, Nr. 35 u. ${ }^{6} 6$.

b) Arch. f. є: p. Pathol. u. Pharmakol. 77, I22. IgI4.

7) Zit. nach JUNGMANN, 32. Kongre $3 \mathrm{f}$. inn. Med. x920, S. 146.

s) Zeitschr. f. Biol. 64 (N. F. 46), 44I. I9r4.

9) Zeitschr. f. Biol. 63 (N. F. 45), 83. I9I4.

10) Ebenda 68 (N. F. 50), 3I. I9I8. 
auch in Verbindung mit der extrarenalen Wasser- und Salzausscheidung und dem intermediären Wasserwechsel. Es braucht nur daran erinnert $z u$ werden, daß die Stelle des Salzstiches in der Medulla oblongata ganz nahe dem Sekretionszentrum der Parotis liegt. Es ist von Interesse, daß die höchste Endstation dieses Regulationsapparates, die wir bisher kennen, das Zwischenhirn, nahe beieinander verschiedene ähnliche vegetative Zentren beherbergt, namentlich ein solches für die Zuckerausscheidung, ferner für die Wärmeregulation [Tuber cinereum, IsENschmID $\left.{ }^{1}\right)$ ], für den Gefäßtonus, die SchweiBsekretion usw. Phylogenetisch ist das Zwischenhirn eine relativ sehr alte Gehirnanlage, die schon bei den niederen Wirbeltieren stark entwickelt ist. Bei den höheren Säugern und namentlich beim Menschen sind den Zwischenhirnzentren wahrscheinlich noch weitere Einrichtungen in den höheren Gebieten des Großhirns übergeordnet.

Es ist leicht, sich auf Grund dieses Tatsachenmaterials eine Vorstellung vom Zustandekommen der, Osmoregulation zu machen, wobei mindestens $4-5$ Stationen übereinanderbzw. ineinandergeschachtelt sind. Die Einzelheiten des Vorgangs mögen zunächst der Phantasie eines jeden überlassen bleiben.

Phylogenetische und ontogenetische Entwicklung der Homoiosmie. Die Eigenschaft, einen bestimmten osmotischen Druck unabhängig von der Umgebung festzuhalten, ist nicht allen Tieren eigen, sondern erscheint an einer bestimmten Stelle der phylogenetischen Reihe. Das läßt sich am schönsten bei den im Wasser lebenden Tieren nachweisen. In den Klassen der Coelenteraten, Würmer, Echinodermen, Mollusken und Arthropoden entspricht der osmotische Druck der Körperflüssigkeiten noch dem des Milieus, in dem sie leben, also dem Meer- oder Süßwasser (poikilosmotische Tiere), wie folgende Tabelle an einigen Beispielen zeigt ${ }^{2}$ ).

Tabelle 1.

\begin{tabular}{|c|c|c|c|}
\hline & & $\triangle$ Blut & $\Delta$ Milieu \\
\hline 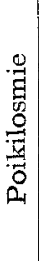 & $\begin{array}{l}\text { Mollusken: } \\
\text { Anodonta cygnea, Teichmuschel } \\
\text { Arthropoden: } \\
\text { Homarus vulgaris, Hummer } \\
\text { Wirbeltiere: } \\
\text { Fische. Selachier } \\
\text { Torpedo marmorata (electrica), } \\
\text { Zitterrochen . . . . . . . . }\end{array}$ & $\begin{array}{l}-0,2^{\circ} \\
-2,29^{\circ}\end{array}$ & $\begin{array}{c}\text { Meerwasser } \\
-2,3^{\circ}\end{array}$ \\
\hline 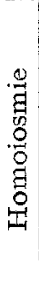 & $\begin{array}{l}\text { Teleostier } \\
\text { Crenilabrus pavo, Lippfisch. } \\
\text { Barbus fluviatilis, Flußbarbe } \\
\text { Amphibien: } \\
\text { Rana esculenta, Wasserfrosch } \\
\text { Saugetiere: } \\
\text { Rind . . . . . . . . . . . } \\
\text { Mensch. . . . . . . . . . }\end{array}$ & $\begin{array}{l}-0,75^{\circ} \\
-0,47^{\circ} \mathrm{bis} \\
-0,56^{\circ} \\
-0,46^{\circ} \\
-0,59^{\circ} \\
-0,56^{\circ}\end{array}$ & $\begin{array}{c}\text { Süßwasser } \\
-0,03^{\circ}\end{array}$ \\
\hline
\end{tabular}

Tabelle 2

Übergangszustand von Poikilosmie zu Homoiosmie:

Pleuronectes platessa, Scholle (Teleostier) an verschiedenen Orten.

\begin{tabular}{c||c|c}
\hline Fangort & $\Delta$ Blut & $\Delta$ Meerwasser \\
\hline \hline Kiel . . . . . . . . & $-0,655^{\circ}$ & $-1,09^{\circ}$ \\
Ostsee . . . . . . & $-0,719^{\circ}$ & $-1,3^{\circ}$ \\
Kattegat . . . . . & $-0,73^{\circ}$ & $-1,66^{\circ}$ \\
Helgoland . . . . & $-0,79^{\circ}$ & $-1,9^{\circ}$
\end{tabular}

Erst innerhalb des Stammes der Wirbeltiere vollzieht sich allmählich der Übergang von der Poikilosmie zur Homoiosmie. Während die Selachier (Elasmobranchier, Plagiostomen), deren inneres Skelett noch aus Knorpel besteht und $\mathbf{z u}_{\mathrm{N}}$ denen

1) Arch. f. experim. Pathol. u. Pharmakol. 76, 202. 1914.

2) $\mathrm{Zu}$ den Tabellen vergleiche: Hamburger, Osmotischer Druck und Ionenlehre. 1902-r904 bei J. F. Bergmann, desgl. I912 Allg. Med. Verl. Anst. - Botazzi, osmot. Druck und elektr. Leitfähigkeit der einzelligen, pflanzlichen und tierischen Organismeo. Ergebn. d. Physiologie Jahrg. 7, I907, S. I6x-402. Höber, Physikal. Chemie d. Zelle u. d. Gewebe, 3. Aufl. rgIr. z. B. die Haifische gehören, den osmotischen Druck des Seewassers besitzen (im Golf von Neapel $\triangle=-2,3^{\circ}$ ) und mit diesem ändern, haben bereits die Teleostier, die Knochenfische, einen osmotischen Druck, der (im gleichen Golf) nur der Hälfte desjenigen des Seewassers entspricht und von der Umgebung ziemlich unabhängig ist. Aber eine völlige Unabhängigkeit ist es auch nicht, denn die Teleostier der Zuidersee, deren Salzgehalt an verschiedenen Stellen sehr ungleich ist, wandern von Gegenden, deren osmotischer Druck ihnen nicht paßt, in Scharen ab. Die Scholle hat schon einen eigenen osmotischen Druck, ändert ihn aber innerhalb gewisser Grenzen entsprechend dem Salzgehalt verschiedener Meeresgegenden (Tabelle 2). Diese Tatsachen dürften zu den Gründen gehören, die überhaupt den getrennten Aufenthalt von Süß- und Seewassertieren bedingen. Erst bei höheren Vertebraten, die neben Kiemen schon eine Lungenatmung besitzen, wird die Konstanz und Unabhängigkeit des osmotischen Druckes eine vollkommene, so daß diese Tiere, auch wenn sie im Wasser leben, den gleichen osmotischen Druck des Blutes besitzen wie ihnen nahestehende Landtiere.

Der osmotische Druck ist also für gewisse höhere Tierklassen eine konstante Größe, deren Aufrechterhaltung für den normalen Ablauf der Lebensvorgänge unumgänglich nötig ist, ähnlich wie die Eigentemperatur, wie der Gehalt an Wasser, Eiweißkörpern, Blutzucker, wie der Partiardruck des Sauerstoffes usw. Dabei ist es interessant, daß diese Konstanten in der Tierreihe keineswegs gleichzeitig auftreten. So besitzen z. B. die Kaltblüter noch keine Eigentemperatur, während der osmotische Druck $z$. B. beim Frosch einen konstanten Wert, entsprechend einer o,6 proz. NaCl-Lösung, aufweist.

Im großen Uberblick geht aber doch die Entwicklung dieser Eigenschaften, die das Lebewesen von seiner Umgebung mehr und mehr loslösen, mit der Ausbildung der entsprechenden Hirnzentren (s. oben) parallel.

Nicht nur phylogenetisch, sondern auch ontogenetisch läßt sich die allmähliche Entwicklung der Homoiosmie und ihrer Störungen dartun. Die Beobachtungen hierüber sind allerdings noch wenig zahlreich. HAGNER ${ }^{1}$ ) hat an der SALGEschen Klinik gezeigt, daß der menschliche Säugling seinen osmotischen Druck nicht mit der gleichen Zähigkeit wie der Erwachsene einzuhalten und gegen Einflüsse verschiedener Ernährung 'sowie andere Einwirkungen aufrechtzuerhalten vermag. Ähnliches wurde an jungen Säugetieren beobachtet. Auch die merkwürdigen Wanderungen mancher Fische dürften sich zum Teil hierdurch erklären. Während der ausgewachsene Lachs vom osmotischen. Druck der Umgebung ziemlich unabhängig ist - denn er kann im FluB wie im Meer leben, - sind seine Eier, die er nur im FluB ablegt, gegen osmotische Einflüsse sehr empfindlich. Bringt man Lachseier in Meerwasser, so gehen sie regelmäßig zugrunde. Umgekehrt verhalten sich wahrscheinlich die Aale, die zum Laichen aus den Flüssen in beträchtliche Meerestiefen von besonders hohem Salzgehalt wandern.

Auch beim erwachsenen Menschen kann, wie schon kurz erwähnt wurde, unter gewissen pathologischen Verhältnissen die Fähigkeit zur Aufrechterhaltung des normalen osmotischen Drucks Einbuße erleiden oder ganz verloren gehen. Das kommt besonders bei Erkrankungen der Nieren vor. Bei chronischen Nierenschädigungen, die mit einer Ausscheidungsstörung und daher mit einer Anhäufung von Stoffwechselprodukten in den Geweben einhergehen, sehen wir allmählich den osmotischen Druck des Blutes etwas ansteigen. Wir finden dann häufig Gefrierpunktswerte von etwa $-0,62^{\circ}$ (statt des Normalwertes von $-0,56^{\circ}$ ). Auf diese mäßige Anderung können sich offenbar die Körperzellen noch umstellen, denn die Lebensfähigkeit bleibt dabei zuweilen noch einige Jahre erhalten. Wenn jedoch die Niereninsuffizienz eine absolute wird oder sehr schnell eintritt, also bei akuter Urämie vom asthenischen Typus, sehen wir eine plötzliche gewaltige Zunahme des osmotischen Drucks, so daß die Gefrierdepression des Blutes bis auf $-0,75^{\circ}$ und noch weiter ansteigt. Daß

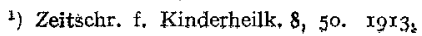


in diesen Fällen der osmotische Regulationsmechanismus des Körpers versagt, geht unter anderem aus folgendem hervor ; ich konnte zeigen, daB bei Urämien solcher Art auch durch ausgiebige Verdünnungsmaßnahmen (Aderlaß, große Wasserzufuhr auf jedem möglichen Wege) die Verminderung der Hyperosmie meist nicht gelingt ${ }^{1}$ ). Die Zellen haben ihre Reaktionsfähigkeit gegen osmotische Reize verloren. Mit der hierdurch bedingten unaufhaltsamen Veränderung des osmotischen Innendrucks ist das menschliche Leben schließlich nicht mehr vereinbar.

Schluß. Wenn wir die Hauptbedeutung des osmotischen Druckes für höhere Organismen in seinem konstanten Niveau erblicken, so soll damit nicht in Abrede gestellt werden, daß Abweichungen vom isosmotischen Punkt unausgesetzt vorkommen, ja da $B$ sie integrierende $Z$ wischenstadien bei vielen komplexen Vorgängen sind. Aber man hat ihre Rolle nicht so zu deuten, daß wie im Membranversuch, ein Druckunterschied physikalisch ausgeglichen wird und daß die hierbei entstehenden statischen Kräfte nun eine sinnreiche Aufgabe erfüllen. Die Kräfte der unbelebten Natur wirken ohne bestimmtes Ziel. Der Wind treibt sein Spiel 'mit Blüten und Blättern, unbekümmert um einen zweckmäßigen Erfolg. Er unterscheidet nicht, wohin der Samen einer männlichen Blüte verweht wird. Nur die sinnreichen Einrichtungen der Pflanze bewirken, daß die wenigen Keime, die zufällig auf eine weibliche Fruchtanlage fallen, einen Reiz vermitteln. Erst hierdurch werden die komplizierten Kräfte feingebauter Zellkonstruktionen ausgelöst,', welche die wunderbaren Erscheinungen der Keimung, des Wachstums, der Reifung vollbringen. Ebenso wirken im Gewebe höherer Tiere $\mathrm{Ab}$ weichungen vom normalen osmotischen Druck gleich vielen ähnlichen Zustandsänderungen als Reiz und veranlassen eine Reaktion. Durch diese werden viel kompliziertere Kräfte, die an die hochorganisierte Struktur der Zelle gebunden sind, in Bewegung gesetzt. Erst hierdurch wird der an sich ziellose osmotische Druckunterschied entweder in eine zweckmäßige physiologische Funktion umgewandelt oder unschädlich gemacht, und der normale Druck, also auch die osmotische Reizschwelle, wiederhergestellt.

Wie eine feine analytische Wage, wie ein kompliziertes Uhrwerk zum tadellosen Funktionieren auf konstante Temperatur und bestimmten Trockenheitsgrad der Umgebung angewiesen ist, so bedarf die Zelle des höheren Tieres zur normalen Erfüllung ihrer vielseitigen Aufgaben eines bestimmten osmotischen Drucks, ebenso wie sie an eine bestimmte Temperatur, Sauerstoffspannung, Wasserstoffionenkonzentration, Oberflächenspannung usw. gebunden ist. Nur niedere Organismen, von denen die Natur geringere Dienste verlangt, können dieser Bedingungen entraten, wie ein grobes Werkzeug den Unbilden der Witterung zu trotzen vermag.

\section{ORIGINALIEN.}

PSEUDOSKLEROSE (WESTPHAL - STRÜMPELL) mit Cornealring (Kayser-Fleischer) und doppelseitiger Scheinkatarakt, die nur bei seitlicher Beleuchtung sichtbar ist und die der nach Verletzung durch Kupfersplitter entstehenden Katarakt ähnlich ist $\left.{ }^{2}\right)$.

$$
\text { Von }
$$

E. Siemerling und $\mathrm{H}$. Oloff.

Aus der Psychiatrischen und Nervenklinik Kiel. (Direktor: Geh. Med.-Rat Prof. Dr. SIEMERLING.)

Die ursprünglich von $\mathrm{C}$. WestPhal als Pseudosklerose Deschriebene und als Neurose aufgefaßte Erkrankung ( 1883 ) hat im Laufe der Jahrzehnte eine interessante Wandlung in der neurologischen Klassifikation durchgemacht. Der Begriff der Neurose ist ganz gefallen. Trotz der oft äußerlich vorhandenen Ähnlichkeit mit der multiplen Sklerose ist sie von dieser Erkrankung weit abgerückt und hat sich mehr der Schüttellähmung und der progressiven lentikulären Degeneration (WILSON) genähert. Sie stellt sich heute dar als eine eigenartige Stoffwechselerkrankung, die wahrscheinlich auf eine juvenile Lebercirrhose aus bisher unbekannter Ursache zurückzuführen ist, mit Rückwirkung auf das Zentralnervensystem und bestimmte Abschnitte des optischen Apparates (wie Cornea).

Der Ausbau der klinischen Symptome hat eine wesentliche Förderung durch StRüMPELls Beobachtungen erfahren. Mit der zugrundeliegenden Lebererkrankung befaßten sich Arbeiten von Vörsch, SALUS, Rumper u. a. I902 brachte die bedeutungsvolle Entdeckung von dem Cornealring durch KAYSER und FLeISCHER: Grünlich brauner Pigmentring in der Peripherie der Cornea in der Descemerschen Membran. Durch die anatomischen Untersuchungen von HössLis und Alzheimer, A. Westphal, Stöcker, Spielmeyer erfuhr das Krankheitsbild eine wesentliche Bereicherung und Erweiterung. Nach Spielmeyers Untersuchungen gibt es bei Pseudosklerose und der WiLsonschen Krankheit keine trennenden

1) Vgl. REISS, Kochsalzstoffwechsel und Wassergehalt des Blatserums. 26. Kongre f. inn. Med. I909, S. I50.

2) Nach einem am r6. Februar Igz2 in der medizinischen Gesellschaft zu Kiel gehaltenen Vortrag mit Vorstellung. Sitzungssber, in der Münch, med, Wochenschr, 1922, Nr. Io S. 390 , histopathologischen Momente, sondern es handelt sich um ein und denselben KrankheitsprozeB.

Die neueren Forschungen über die nach Grippe und Encephalitis entstehenden Folgeerkrankungen des Zentralnervensystens mit dem Charakter des amyostatischen Symptomenkomplexes (STRÜMPELL) oder des dystonischen Syndroms (STERTZ) und den dabei erhobenen anatomischen Befunden werfen ein interessantes Licht auch auf die Pseudosklerose.

Der Kranke, um den es sich handelt, befindet sich schon seit r 917 in poliklinischer Beobachtung (Prof. RuNGE). 43 jähriger Arbeiter. Die Mutter hat $4 \mathrm{mal}$ an Ikterus gelitten, ein Bruder an Veitstanz. Im Alter von IO-I2 Jahren hatte er Ikterus. Keine luische Infektion. Früher war er 7 Jahre Brauereiböttcher und hat damals täglich 4 l Bier getrunken. Jetzt kein Alkoholabusus. Starker Raucher. 1916 Beginn des Zitterns in den Händen. 1917 Zittern des Kopfes, Zittern der Hände, besonders rechts allmähliche Zunahme des Zitterns. Status: im Februar 1922. Starkes Wackeln und Schütteln des Kopfes, I20 Oszillationen in der Minute. Bei extremen Stellungen des Kopfes, besonders beim Beugen nach rückwärts hört das Wackeln auf, ebenso in völliger Ruhelage des Kopfes. Starrer Gesichtsausdruck. Sprache langsam, aber nicht artikulatorisch gestört. Schilddrüse nicht vergrößert. An den Armen in der Ruhe leichtes Zittern des ganzen Armes, bei Bewegungen z.B. nachVorwärtsstrecken derArme starke Zunahme des Schüttelns, förmliches Flügelschlagen und Schwimmbewegungen (STRÜMPELL). Stoßende Zuckungen im Pectoralis major. Bei komplizierten Bewegungen, z. B. Auskleiden, Essen, Schreiben, sehr starkes Schütteln. In den unteren Extremitäten viel geringeres Zittern. Kein Romberg. Keine Pro- und Retropulsion. Hypotonie an oberen und unteren Extremitäten sehr ausgesprochen. Abdominalreflexe fehlen. Sehnenreflexe gut erhalten. Im Blutbild nichts besonderes. WaR. im Blut negativ. An der Haut keine Acne, keine besondere Pigmentierung. Psychisch: gleichmäBige, ruhige Stimmung. Geht seiner Beschäftigung nach. Keine Demenz. Lumbalpunktion: Druck 90-100. Zellen 17, Nonne positiv. Herr Prof. SchittexHELM und Herr Dr. HARPUDER hatten die Freundlichkeit in der medizinischen Klinik eine Untersuchung vorzunehmen und danke ich beiden bestens. Aus dem Befund ist folgendes zu erwähnen: Herz: Grenzen nicht erweitert, lk. I Querfingerbreit innerhalb der Medioclavicularlinie.

Töne: An der Herzspitze bei Exspirationsstellung ein leises akzidentelles Geräusch während der Systole, das bei Inspirationsstellung nicht $\mathrm{zu}$ hören ist. Keine besondere Akzentuation.

Leib: weich, eindrückbar, keine Resistenzen, kein Druckschmerz. Leberrand querfingerbreit unterhalb des Rippenbogens fühlbar. Milz nicht zu tasten. 